\title{
CORRECTION
}

\section{Correction to: In vivo Cell Tracking Using Non-invasive Imaging of Iron Oxide-Based Particles with Particular Relevance for Stem Cell-Based Treatments of Neurological and Cardiac Disease}

Joel C. Glover $\odot,{ }^{1,2}$ Markus Aswendt, ${ }^{3}$ Jean-Luc Boulland, ${ }^{1,2}$ Jasna Lojk, ${ }^{4}$ Stefan Stamenković, ${ }^{5}$ Pavle Andjus, ${ }^{5}$ Fabrizio Fiori, ${ }^{6}$ Mathias Hoehn, ${ }^{3}$ Dinko Mitrecic, ${ }^{7}$ Mojca Pavlin, ${ }^{4,8}$ Stefano Cavalli, ${ }^{9}$ Caterina Frati, ${ }^{9}$ Federico Quaini, ${ }^{9}$ on behalf of the EU COST Action 16122 (BIONECA)

\footnotetext{
${ }^{1}$ Laboratory for Neural Development and Optical Recording (NDEVOR), Department of Molecular Medicine, Institute of Basic Medical Sciences, University of Oslo, PB 1105, Blindern, Oslo, Norway

${ }^{2}$ Norwegian Center for Stem Cell Research, Oslo University Hospital, Oslo, Norway

${ }^{3}$ Institut für Neurowissenschaften und Medizin, Forschungszentrum Jülich, Leo-Brandt-Str. 5, 52425, Jülich, Germany

${ }^{4}$ Group for Nano and Biotechnological Applications, Faculty of Electrical Engineering, University of Ljubljana, Trzaska cesta 25, Ljubljana, Slovenia

${ }^{5}$ Center for Laser Microscopy, Department of Physiology and Biochemistry, Faculty of Biology, University of Belgrade, PB 52, Belgrade, 10001, Serbia

${ }^{6}$ Department of Applied Physics, Università Politecnica delle Marche - Di.S.C.O., Via Brecce Bianche, 60131, Ancona, Italy

${ }^{7}$ Laboratory for Stem Cells, Croatian Institute for Brain Research, School of Medicine, University of Zagreb, Zagreb, Croatia

${ }^{8}$ Institute of Biophysics, Faculty of Medicine, University of Ljubljana, Vrazov trg 2, Ljubljana, Slovenia

${ }^{9}$ Department of Medicine and Surgery, University of Parma, Parma, Italy
}

Correction to: Mol Imaging Biol (2020) 22:1469-1488 https://doi.org/10.1007/s11307-019-01440-4

This article was updated to correct numerous mismatched references.

Publisher's Note Springer Nature remains neutral with regard to jurisdictional claims in published maps and institutional affiliations. 